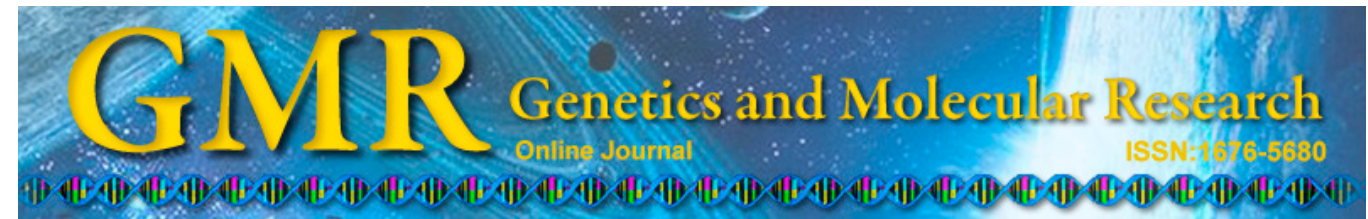

\title{
Correlation of MSH3 polymorphisms with response and survival in advanced non-small cell lung cancer patients treated with first-line platinum-based chemotherapy
}

\author{
X.-L. Xu ${ }^{1,2 *}$, Y.-L. Yao ${ }^{1 *}$, W.-Z. Xü ${ }^{2}$ J.-G. Feng ${ }^{1}$ and W.-M. Mao ${ }^{1,2}$ \\ ${ }^{1}$ Key Laboratory on Diagnosis and Treatment Technology for Thoracic Cancer, \\ Zhejiang Cancer Hospital (Zhejiang Cancer Research Institute), \\ Hangzhou, Zhejiang Province, China \\ ${ }^{2}$ Department of Medical Oncology, Zhejiang Cancer Hospital, \\ Hangzhou City, China \\ *These authors contributed equally to the study. \\ Corresponding author: W.-M. Mao \\ E-mail: maowm1318@163.com
}

Genet. Mol. Res. 14 (2): 3525-3533 (2015)

Received July 21, 2014

Accepted October 7, 2014

Published April 15, 2015

DOI http://dx.doi.org/10.4238/2015.April.15.16

\begin{abstract}
Mismatch repair (MMR) genes, as well as the nucleotide excision repair genes, play an important role in removing cisplatinDNA adducts, and the mutation of MMR genes in tumors can lead to a decreased response to platinum-based therapies. We examined MutS homolog 3 (MSH3), a mismatch repair gene, and whether polymorphisms of MSH3 were associated with response and survival in advanced non-small cell lung cancer (NCSLC) patients who were treated with platinum-based chemotherapy. The peripheral blood of 180 advanced NCSLC patients who were treated with first-line platinum-based chemotherapy was collected to determine the patients' genotypes of MSH3. The three genotypes of the MSH3 polymorphisms rs26279, rs1650697 and rs1105524 were investigated. A statistically significant association was observed between the polymorphism
\end{abstract}


rs26279 (Ala1054Thr) and sensitivity to platinum-based chemotherapy $(\mathrm{P}=0.014)$. A significant correlation was found between $\mathrm{rs} 1105524$ and progression-free survival (PFS), with the G/A and A/A genotypes (median survival time: 14.27 months; $95 \% \mathrm{CI}=9.80-18.75$ ) suffering shorter survival than patients with the $\mathrm{G} / \mathrm{G}$ genotype (median survival time: 26.37 months; $95 \% \mathrm{CI}=15.03-37.71)(\mathrm{P}=0.04)$. Our results showed that single nucleotide polymorphisms in MSH3 had an impact on the chemotherapy response and prognosis of advanced NCSLC patients who were treated with platinum-based chemotherapy.

Key words: MutS homolog 3; Single nucleotide polymorphism; Platinum-based chemotherapy; Non-small cell lung cancer

\section{INTRODUCTION}

According to the GLOBOCAN 2012 database (Ferlay et al., 2012), lung cancer is the most common cancer in the world and the most common cause of cancer-related death (1.59 million deaths, $19.4 \%$ of the total), with more than $58 \%$ of the deaths occurring in less developed regions. Because of its high fatality (the overall ratio of mortality to incidence is 0.87 ), large numbers of studies focus on the prevention and treatment of lung cancer. Non-small cell lung cancer (NSCLC) accounts for approximately $85 \%$ of all lung cancer cases (Xie et al., 2012). In NSCLC, almost half of the patients have advanced and metastatic disease and have missed the opportunity to have surgery. Since platinum-based chemotherapy provides modest survival benefits, a predictive marker for diagnostic and chemotherapeutic outcome is needed.

The main activity of cisplatin (DDP) is the formation of DDP-DNA adducts, which block replication and inhibit transcription. Removal of these adducts is mainly carried out by the nucleotide excision repair system. Postreplication mismatch repair (MMR) is composed of several proteins including MutS homolog 3 (MSH3), whose function is to eliminate replication errors and maintain genomic stability. MMR genes play an important role in removing DDP-DNA adducts and mutation in MMR genes may lead to a decreased response to platinum-based chemotherapy (Conde-Pérezprina et al., 2012). The MSH3 gene is located on chromosome 5q11-13 and was first described in 1989 (Hirata et al., 2008); somatic mutation of MSH3 occurs frequently in MMR-deficient cancers, such as colon carcinoma (Takahashi et al., 2011; Park et al., 2013), lung cancer, breast cancer (Conde et al., 2009) and oral squamous cell carcinoma (Mondal et al., 2013). The association of MSH3 gene polymorphisms in ovarian cancer has also been reported, which suggests that MSH3 polymorphism may be a risk factor for ovarian cancer (Song et al., 2006). Hirata et al. (2008) investigated MMR polymorphisms in sporadic prostate cancer. They found evidence for an increased risk of sporadic prostate cancer with single nucleotide polymorphisms (SNPs) in the MSH3 variants rs1805355 and rs26279. However, the relationship between MSH3 polymorphisms and advanced NSCLC patients treated with chemotherapy has not been studied. Different activities and functions of these genes as well as SNP variations may modulate repair capacity and contribute to individual variations in platinum-based chemotherapy responses.

These reports prompted us to investigate MSH polymorphisms rs1105524, rs 1650697 and rs26279 and their association with chemotherapy sensitivity and survival in advanced NSCLC patients treated with platinum-based chemotherapy. 


\section{MATERIAL AND METHODS}

\section{Patients and methods}

Patient selection criteria: eligible patients had histologically or cytologically confirmed stage IIIB or IV NSCLC and they also met the following criteria: 1) Age between 18 and 80 years; 2) Eastern Cooperative Oncology Group (ECOG) performance status of 3 or less; 3) One or more measurable or assessable lesions; 4) Life expectancy of more than 4 weeks; 5) No previous chemotherapy or radiotherapy. The main exclusion criteria were concomitant malignancy or a second primary malignancy within the last 5 years.

\section{Chemotherapy regimens and clinical response evaluation}

To facilitate the interpretation of the results, all chemotherapy regimens were classified into the following three groups: 1) gemcitabine (GEM) plus DDP/carboplatin (CBP), Navelbine (NVB) plus DDP/CBP regimens, 2) taxol (TAX)/docetaxel (DOC) plus DDP/CBP, and 3) DDP/ CBP plus others agents. All patients received platinum-based chemotherapy: 114 patients received intravenous doses of DDP $\left(75 \mathrm{mg} / \mathrm{m}^{2}\right)$ or CBP area under the curve (AUC) $(5 \mathrm{mg} / \mathrm{mL})$ per min on day 1 plus gemcitabine $\left(1000 \mathrm{mg} / \mathrm{m}^{2}\right)$ on days 1 and 8 every 3 weeks. Thirty patients received intravenous doses of DDP/CBP on day 1 plus taxol $\left(175 \mathrm{mg} / \mathrm{m}^{2}\right)$ on day 1 and DOC $\left(75 \mathrm{mg} / \mathrm{m}^{2}\right)$ on day 1 , and 24 patients received intravenous doses of DDP/CBP on day 1 plus others agents.

According to the Response Evaluation Criteria in Solid Tumors (RECIST) (Therasse et al., 2000), tumor responses can be categorized as complete response (CR), partial response (PR), stable disease (SD) and progressive disease (PD). All responses were evaluated on the basis of change in lesion size derived from a computed tomography scan at least 6 weeks after the initial assessment. Written informed consent was obtained from all patients enrolled in this study.

\section{DNA extraction and genotyping}

DNA was successfully extracted from the blood of all patients, and more than $95 \%$ of DNA samples were successfully genotyped for all candidate SNPs. The DNA was amplified by multiplex polymerase chain reaction (PCR) and multiplex PCR primers were used. Then, the multiple PCR products were used for a series of SNaPshot multiplex single base extension reactions, which were carried out by using the SNaPshot Multiplex Kit (Applied Biosystems Co., Ltd., Foster City, CA, USA); the extension products were sequenced with an ABI3730XL DNA analyzer.

\section{Statistical analysis}

Statistical analysis was performed with the SPSS standard version 17.0 (SPSS Inc., Chicago, IL, USA). Demographic and clinicopathological characteristics were compared across the genotypes by using the chi-square test. The association between the MSH3 polymorphism and clinical response was also assessed by using the chi-square test. The univariate analyses were analyzed by using the Kaplan-Meier method and the log-rank test, and a Cox multivariate analysis (likelihood backwards method) was used to calculate the independent relative risks for progression-free survival (PFS) and overall survival (OS). Differences were considered significant when $\mathrm{P}<0.05$. 


\section{RESULTS}

\section{Genotype information}

The three genotypes of MSH3 polymorphisms rs26279, rs1650697 and rs1105524 were investigated. The distribution of the genotypes for the three MSH3 polymorphisms and the allelic frequencies is shown in Table 1. For MSH3 rs1105524, the A/A genotype was found in 103 patients (57.2\%), the G/A in 67 (37.2\%), and the remaining $10(5.6 \%)$ patients had the $\mathrm{G} / \mathrm{G}$ genotype. The genotype $\mathrm{G} / \mathrm{G}$ of the rs 1650697 polymorphism had a frequency of $44.4 \%$, whereas the heterozygous $\mathrm{G} / \mathrm{A}$ and homozygous $\mathrm{A} / \mathrm{A}$ variants had a frequency of 37.8 and $17.8 \%$, respectively. The frequencies of the $\mathrm{A} / \mathrm{A}, \mathrm{G} / \mathrm{A}$, and G/G genotypes of MSH3 rs 26279 were 41.1, 38.9, and 20\%, respectively. All genotype distribution was compatible with the Hardy-Weinberg principle of equilibrium.

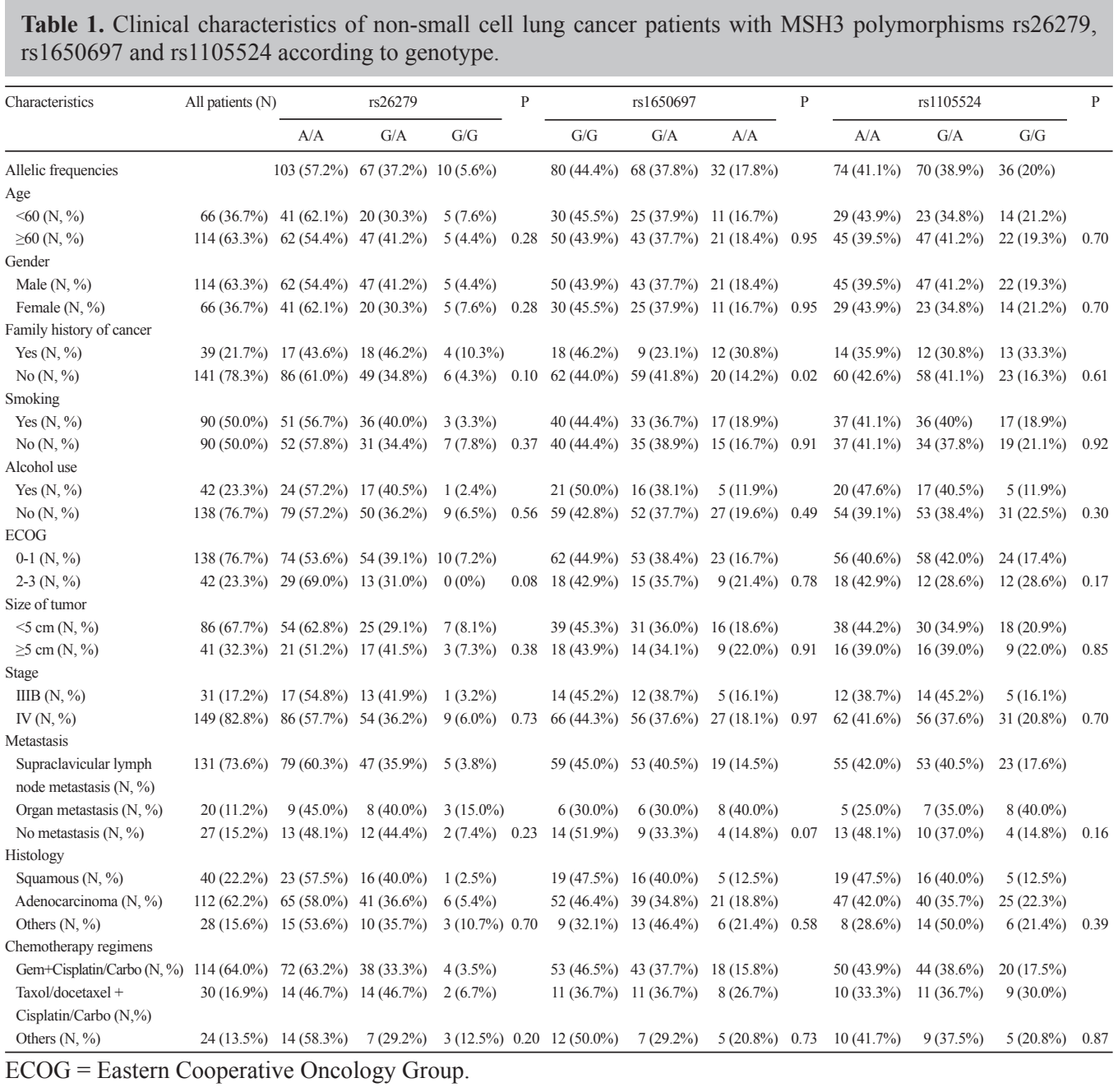




\section{Patient characteristics and MSH3 genotypes}

Association between the MSH3 genotypes and the clinical characteristics of patients with advanced NSCLC are respectively shown in Table 1 . To estimate the relative risk of NSCLC associated with each MSH3 genotype, the following factors were calculated: age, gender, family history of cancer, history of smoking and alcohol use, histology of tumor, ECOG, size of tumor, stage, metastasis, and radiotherapy and chemotherapy regimens. A total of 180 patients with advanced NSCLC were recruited into this study, of which 114 were men and 66 were women. The mean age of patients was 57 years (ranging from 35 to 78 years old). Thirty-nine $(21.7 \%)$ patients had a family history of cancer and $141(78.3 \%)$ patients did not. Half were smokers and 39 (23.3\%) patients had a history of alcohol use. Staging was evaluated by using the 7th edition of the American Joint Committee on Cancer Tumor-Node-Metastasis (TNM) staging system. Of the 180 patients, $31(17.2 \%)$ had stage IIIB disease and $149(82.8 \%)$ had stage IV disease. A single-factor analysis of the MSH3 genotypes showed that family history of cancer was the only significant variable for the rs 1650697 genotype $(\mathrm{P}=0.02)$.

\section{Gene polymorphisms and clinical response}

A statistically significant association was observed between the polymorphism rs26279 (Ala1054Thr) and sensitivity to platinum-based chemotherapy $(\mathrm{P}=0.014$, Table 2). However, there were no significant correlations between MSH3 rs1650697 or rs1105524 and objective response.

\begin{tabular}{|c|c|c|c|c|}
\hline MSH3 phenotype & PR & SD & PD & $P$ \\
\hline \multicolumn{5}{|l|}{ rs 26279} \\
\hline $\mathrm{A} / \mathrm{A}$ & $38(36.9 \%)$ & $52(50.5 \%)$ & $13(12.6 \%)$ & \\
\hline $\mathrm{G} / \mathrm{A}$ & $38(56.7 \%)$ & $26(38.8 \%)$ & $3(4.5 \%)$ & \\
\hline $\mathrm{G} / \mathrm{G}$ & $1(10 \%)$ & $7(70 \%)$ & $2(20 \%)$ & 0.014 \\
\hline \multicolumn{5}{|l|}{ rs1650697 } \\
\hline $\mathrm{G} / \mathrm{G}$ & $37(46.3 \%)$ & $35(45 \%)$ & $7(8.7 \%)$ & \\
\hline $\mathrm{G} / \mathrm{A}$ & $33(48.5 \%)$ & $29(42.6 \%)$ & $6(8.9 \%)$ & \\
\hline $\mathrm{A} / \mathrm{A}$ & $7(21.9 \%)$ & $20(62.5 \%)$ & $5(15.6 \%)$ & 0.125 \\
\hline \multicolumn{5}{|l|}{ rs1105524 } \\
\hline $\mathrm{A} / \mathrm{A}$ & $36(48.6 \%)$ & $32(43.2 \%)$ & $6(8.2 \%)$ & \\
\hline $\mathrm{G} / \mathrm{A}$ & $32(45.7 \%)$ & $31(44.3 \%)$ & $7(10 \%)$ & \\
\hline $\mathrm{G} / \mathrm{G}$ & $9(25 \%)$ & $22(61.1 \%)$ & $5(13.9 \%)$ & 0.195 \\
\hline
\end{tabular}

$\mathrm{PR}=$ partial response; $\mathrm{SD}=$ stable disease; $\mathrm{PD}=$ progressive disease. Bold means $\mathrm{P}<0.05$.

\section{Association of genotype and survival}

The correlation between the three MSH3 genotypes and survival was analyzed by using Kaplan-Meier survival curves (Figures 1 and 2). We were unable to obtain survival information for 2 of the 180 patients. A significant correlation was found between rs 1105524 and PFS, with the G/A and A/A genotypes (median survival time: 14.27 months; $95 \%$ confidence interval: 9.80-18.75) suffering shorter survival than patients with the $G / G$ genotype (median survival time: 26.37 months; 95\% confidence interval: 15.03-37.71) $(\mathrm{P}=0.04)$. In the 
multivariate analysis, the MSH3 rs 1105524 polymorphism also served as an independent protective factor for PFS (hazard ratio: $0.329 ; 95 \%$ confidence interval: $0.166-0.653 ; \mathrm{P}=0.00$ ). The PFS for rs 1650697 showed no significant differences in the univariate analysis $(\mathrm{P}=0.10)$. There was also no significant difference in PFS found in the genotypes of rs26279, although patients with the G/A genotype had a slightly shorter PFS (median survival time: 14.0 vs 17.03 vs 18.57 months). However, no significant differences were observed in OS among the three MSH3 genotypes in either the univariate analysis or the multivariate analysis.
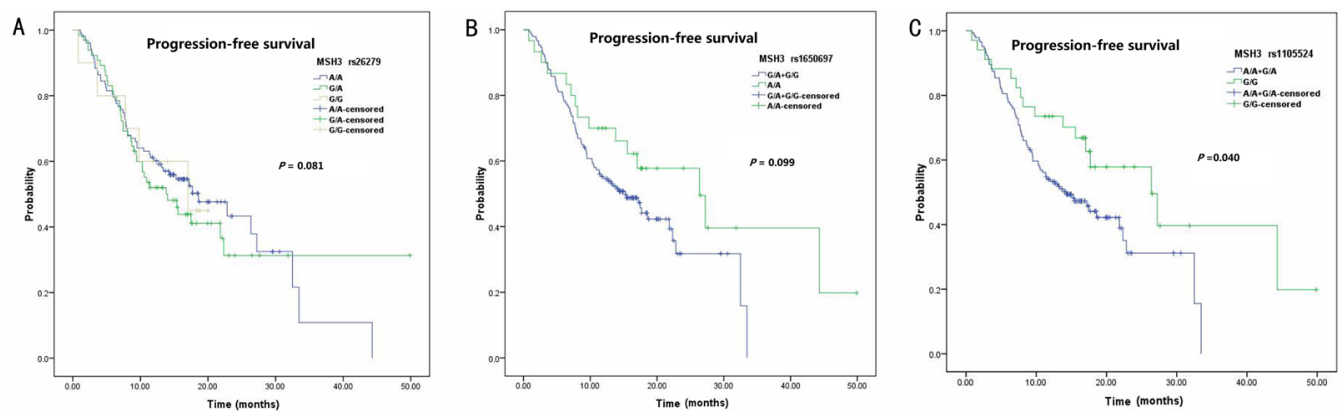

Figure 1. Kaplan-Meier curves of progression-free survival for advanced non-small cell lung cancer patients with different MSH3 polymorphisms: rs26279 (A), rs1650697 (B) and rs1105524 (C).
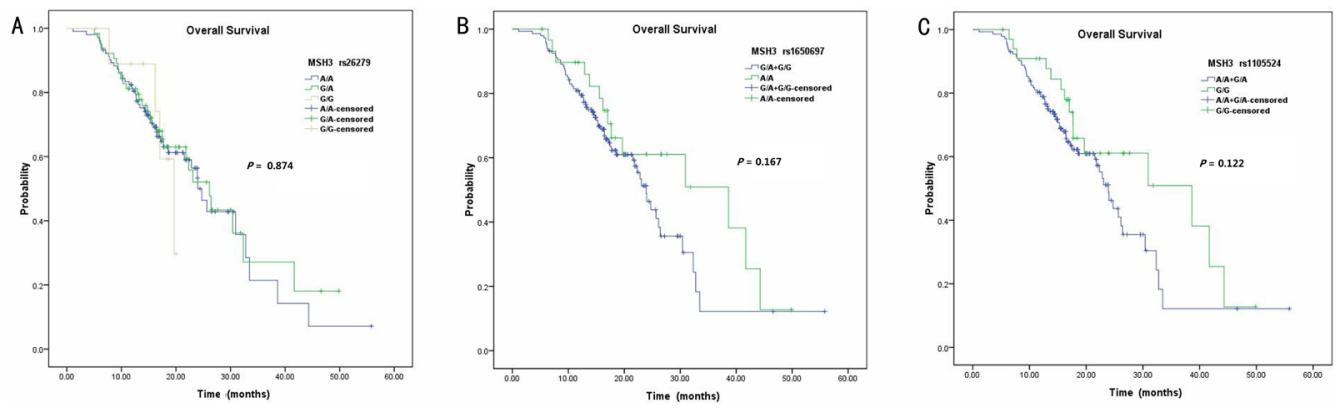

Figure 2. Kaplan-Meier curves of overall survival for advanced non-small cell lung cancer patients with different MSH3 polymorphisms: rs26279 (A), rs1650697 (B) and rs1105524 (C).

\section{DISCUSSION}

There are several partly overlapping DNA repair pathways, including base excision repair (BER), nucleotide excision repair (NER), double strand break repair (DSBR) and MMR. To date, many studies have addressed whether specific SNPs of DNA repair genes impact the effectiveness of platinum-based chemotherapy in NSCLC patients. XRCC1 belongs to the base excision repair (BER) pathway. Previous data suggests that genetic polymorphisms in XRCC1 (Arg399Gln) may be important prognostic factors in platinum-treated patients with advanced NSCLC (Gurubhagavatula et al., 2004). Wei et al. (2011) confirmed the prognostic role of XRCC1 (Arg399Gln) polymorphism in advanced NSCLC. However, it was shown to be a non-specific predictive factor of DDP efficiency. However, it was not shown to be a specific predictive factor of DDP efficiency. 
MMR is also an important pathway for DNA repair. DNA mismatch repair plays a key role in maintaining genomic stability through a highly conserved biological pathway. The specificity of MMR is primarily for base-base mismatches and insertion/deletion mispairs generated during DNA replication and recombination in eukaryotes (Harrington and Kolodner, 2007). Defects in MMR are also associated with genome-wide instability, predisposition to certain types of cancer including hereditary non-polyposis colorectal cancer, and abnormalities in meiosis (Li, 2008). Interestingly, recent studies suggest that MMR-deficient cells are resistant to certain chemotherapeutic drugs such as DDP, which has significant impacts on cancer treatments. MMR participation in non-productive DDP interstrand cross-link (ICL) processing is downstream of BER processing and dependent on Pol $\beta$ misincorporation at DDP ICL sites, which results in persistent DDP ICLs and sensitivity to DDP (Kothandapani et al., 2013). The MSH3 gene is one of the DNA mismatch repair genes that forms the MutS $\beta$ heteroduplex, together with MSH2. The MSH2/MSH3 heterodimer is an ATPase that plays a critical role in mismatch recognition and initiation of repair. It binds to the DNA mismatch by recognizing 2-13-bp insertion-deletion loops. Furthermore, the MSH2/MSH3 heterodimer can also recognize ICLs generated by DNA cross-linkers such as DDP. In addition, MutS $\beta$ also binds to DDP-induced ICLs together with PARP-1, DNA ligase III, XRCC-1, Ku80 and Ku70, suggesting that MutS $\beta$ may also cooperate with other repair pathways to recognize and repair platinum drug-induced ICLs (Zhu and Lippard, 2009). Furthermore, Takahashi et al. (2011) reported that MSH3 can mediate the sensitization of colorectal cancer cells to DDP.

To our knowledge, this is the first comprehensive study to evaluate the association between candidate SNPs of MSH3 genotypes and chemotherapy sensitivity and survival in advanced NSCLC patients. Because a subgroup of patients do not benefit from chemotherapy, the National Comprehensive Cancer Network guidelines pay more attention to individualized diagnosis and treatment. Moreover, identification of predictive markers is most warranted for use in clinical diagnosis, chemotherapy treatment planning and prognosis. Polymorphic variants in DNA repair genes can explain interindividual differences in the response or survival of NSCLC patients independent of their performance status, which is the primary clinical prognostic factor.

Our results showed that patients with the heterozygous G/A genotypes in the three MSH3 polymorphisms displayed a much shorter PFS than homozygous A/A patients or G/G patients. Therefore an MSH3 polymorphism can be considered a risk factor for advanced NSCLC patients treated with DDP-based chemotherapy.

The different MSH3 polymorphisms have a respectively different effect on chemotherapy sensitivity and survival. The rs 26279 polymorphism contains a $\mathrm{G}$ to A substitution in position 3213 of the mRNA that gives rise to an Ala to Thr substitution at position 1045. Vogelsang et al. (2012) reported that the MSH3 rs26279 G/G versus A/A or G/A genotype was positively associated with esophageal cancer. Hirata et al. (2008) found that the G/G or G/A genotype of the MSH3 rs26279 polymorphism might be a risk factor for sporadic prostate cancer. Moreover, the genetic variant of the MSH3 rs26279/MSH6 Gly39Glu - AA/ TC was associated with a decreased risk for breast cancer (Conde et al., 2009). Although the function of this polymorphism is unknown, our data also revealed that NSCLC patients with the $\mathrm{G} / \mathrm{G}$ genotype had a decreased response to platinum-based treatments. In addition, an association between MSH3 rs1650697 and radiation sensitivity of the lungs was investigated, but the results were discarded because of their unreliability (Kelsey et al., 2013). In our study, polymorphism rs1650697 of MSH3 appears to be involved in a family history of cancer, as 
patients with G/G genotypes are susceptible to NSCLC and considered a high risk population (Table 1). Another SNP, rs1105524, has barely been studied before, and interestingly, the genotypes of rs1105524 had a significant impact on PFS in advanced NSCLC patients who were treated with platinum-based chemotherapy. The multivariate analysis, which was corrected for possible confounding factors (radiotherapy, etc.), also validated the impact of the rs 1105524 genotypes on PFS in our population.

However, we acknowledge there were some limitations in our study. First, the small number patients and the data from a single-hospital retrospective study might be due to chance. Thus, multicenter and prospective studies are needed to further assess the prognostic value of MSH3 SNPs in advanced NSCLC. Given the number of patients with available data, we were constrained to use a candidate gene approach as opposed to a genome-wide association study to perform our analysis.

In summary, it is possible that SNPs in MSH3 may modulate DNA repair capacity and contribute to individual variations in chemotherapy response and prognosis. Further studies are needed to determine the molecular and cellular mechanisms underlying the significance of MSH3 SNPs in NSCLC.

\section{Conflicts of interest}

The authors declare no conflict of interest.

\section{ACKNOWLEDGMENTS}

Research supported by the Province Important Technology and Science Program (Special Feature of Major Province Scientific and Technological Program 2011), \#2011C13039-1, \#20112014, and the establishment and NSFC general program, \#81172081, \#2012.01-2015.12.

\section{REFERENCES}

Conde J, Silva SN, Azevedo AP, Teixeira V, et al. (2009). Association of common variants in mismatch repair genes and breast cancer susceptibility: a multigene study. BMC Cancer 9: 344 .

Conde-Pérezprina JC, Léon-Galván MÁ and Konigsberg M (2012). DNA mismatch repair system: repercussions in cellular homeostasis and relationship with aging. Oxid. Med. Cell. Longev. 2012:728430.

Ferlay J, Soerjomataram I, Ervik M, Dikshit R, et al. (2012). GLOBOCAN 2012 v1.0, Cancer Incidence and Mortality Worldwide: IARC CancerBase No. 11.

Gurubhagavatula S, Liu G, Park S, Zhou W, et al. (2004). XPD and XRCC1 genetic polymorphisms are prognostic factors in advanced non-small-cell lung cancer patients treated with platinum chemotherapy. J. Clin. Oncol. 22: 2594-2601.

Harrington JM and Kolodner RD (2007). Saccharomyces cerevisiae Msh2-Msh3 acts in repair of base-base mispairs. Mol. Cell. Biol. 27: 6546-6554.

Hirata H, Hinoda Y, Kawamoto K, Kikuno N, et al. (2008). Mismatch repair gene MSH3 polymorphism is associated with the risk of sporadic prostate cancer. J. Urol. 179: 2020-2024.

Kelsey CR, Jackson IL, Langdon S, Owzar K, et al. (2013). Analysis of single nucleotide polymorphisms and radiation sensitivity of the lung assessed with an objective radiologic endpoin. Clin. Lung Cancer 14: 267-274.

Kothandapani A, Sawant A, Dangeti VS, Sobol RW, et al. (2013). Epistatic role of base excision repair and mismatch repair pathways in mediating cisplatin cytotoxicity. Nucleic Acids Res. 41: 7332-7343.

Li GM (2008). Mechanisms and functions of DNA mismatch pair. Cell Res. 18: 85-98.

Mondal P, Datta S, Maiti GP, Baral A, et al. (2013). Comprehensive SNP scan of DNA repair and DNA damage response genes reveal multiple susceptibility loci conferring risk to tobacco associated leukoplakia and oral cancer. PLoS One 8: e56952. 
Park JM, Huang S, Tougeron D and Sinicrope FA (2013). MSH3 mismatch repair protein regulates sensitivity to cytotoxic drugs and a histone deacetylase inhibitor in human colon carcinoma cells. PLoS One 8: e65369.

Song H, Ramus SJ, Quaye L, DiCioccio RA, et al. (2006). Common variants in mismatch repair genes and risk of invasive ovarian cancer. Carcinogenesis 27: 2235-2242.

Takahashi M, Koi M, Balaguer F, Boland CR, et al. (2011). MSH3 mediates sensitization of colorectal cancer cells to cisplatin, oxaliplatin, and a poly(ADP-ribose) polymerase inhibitor. J. Biol. Chem. 286: 12157-12165.

Therasse P, Arbuck SG, Eisenhauer EA, Wanders J, et al. (2000). New guidelines to evaluate the response to treatment in solid tumors. European Organization for Research and Treatment of Cancer, National Cancer Institute of the United States, National Cancer Institute of Canada. J. Natl. Cancer Inst. 92: 205-216.

Vogelsang M, Wang Y, Veber N, Mwapagha LM, et al. (2012). The cumulative effects of polymorphisms in the DNA mismatch repair genes and tobacco smoking in oesophageal cancer risk. PLoS One 7: e36962.

Wei SZ, Zhan P, Shi MQ, Shi Y, et al. (2011). Predictive value of ERCC1 and XPD polymorphism in patients with advanced non-small cell lung cancer receiving platinum-based chemotherapy: a systematic review and meta-analysis. Med. Oncol. 28: 315-321.

Xie FJ, Zhao P, Kou JY, Hong W, et al. (2012). The T393C polymorphism of GNAS1 as a predictor for chemotherapy sensitivity and survival in advanced non-small-cell lung cancer patients treated with gemcitabine plus platinum. Cancer Chemother. Pharmacol. 69: 1443-1448.

Zhu G and Lippard SJ (2009). Photoaffinity labeling reveals nuclear proteins that uniquely recognize cisplatin-DNA interstrand cross-links. Biochemistry 48: 4916-4925. 